\title{
High-level expression of CXCL1/GROa in uterine cervix cancer is linked to advanced stage and facilitates tumor cell malignant processes in an autocrine/paracrine manner
}

\section{XiaXia Man}

The First Hospital of Jilin University

\section{XiaoLin Yang}

The First Hospital of Jilin University

\section{ZhenTong Wei}

The First Hospital of Jilin University

\section{YuYing Tan}

The First Hospital of Jilin University

WanYing Li

The First Hospital of Jilin University

HongJuan Jin ( $\sim$ kim20150919@163.com )

The First Hospital of Jilin University

\section{Baogang Wang ( $\sim$ baogangwang2004@163.com )}

The First Hospital of Jilin University https://orcid.org/0000-0001-6408-8185

\section{Primary research}

Keywords: Uterine cervix cancer; CXCL1; Malignant behavior; Extracellular regulated protein kinases

Posted Date: May 6th, 2020

DOI: https://doi.org/10.21203/rs.3.rs-25877/v1

License: (9) (i) This work is licensed under a Creative Commons Attribution 4.0 International License. Read Full License 


\section{Abstract \\ Background}

It has previously accepted that several types of human cancer constitutively express CXCL1, which may implicated in various aspects of tumors. Here, we sought to assess the expression and of CXCL1 in human uterine cervix cancer (UCC) and its potential role and mechanism in UCC.

\section{Methods}

CXCL1 protein expression in a uterine cervical tissue microarray was assessed by immunohistochemistry. The roles of CXCL1 on HeLa UCC cells were detected by CCK-8, transwell and apoptosis assays. Western blotting and ERK1/2 blocker PD98059 were utilized to explore whether ERK signal was implicated in CXCL1-mediated UCC processes.

\section{Results}

CXCL1 was expressed by HeLa, PHM1-41 cells as well as cervical tissues, in which UCC tissues showed an obviously high level of CXCL1 compared with non-cancerous tissues. Additionally, of the cancer tissues, CXCL1 high-level expression was notably relevant to poor clinical stages. In vitro, the growth and migration of HeLa cells were enhanced in the presence of exogenous CXCL1. Gain-function assays revealed that CXCL1 overexpression promoted growth and migration response to HeLa cells via autocrine/paracrine manners. Moreover, CXCL1 also led to the inhibition of apoptosis in HeLa cells. Finally, CXCL1 overexpression in HeLa cells influenced the expression of ERK signal-related genes including ERK, p-ERK, cyclin D1 and Bax, and cell malignant behaviors derived from CXCL1 overexpresion were further interrupt in the presence of PD98059.

\section{Conclusion}

Our findings provided the potential roles of CXCL1 as a promoter and the novel understanding of the functional relation of CXCL1 with ERK signal pathway in UCC.

\section{Background}

Uterine cervix cancer (UCC), the common malignancy, represents the fourth most frequently occurring cancer and the fourth leading cause of cancer-related death in females worldwide [1]. It is estimated that there were 57,000 new diagnosed cases of UCC in 2018, with 311,000 patients succumbing to this disease [1]. To date, several etiologies, including human papillomavirus (HPV) infection, immunosuppression (particularly HIV infection), alcohol consumption, smoking, parity, number of sexual partners as well as oral intake of contraceptives, have been shown as predominant risk factors for the cause of 
cervical cancer, in which persistent infection with HPV is the most important component and largely increases the incidence of UCC [2-3]. The persistence of inflammation among women with HPV infection was responsible for malignant transformation of the cervical epithelium [3], suggesting cancer-related flammatory reaction is predominantly involved in the pathogenesis of UCC.

Chemokines, or chemoattractant cytokines, are categorized into four subfamilies, namely CXC, CC, C and $\mathrm{CX} 3 \mathrm{C}$, on the basis of the number and spacing of the conserved cysteine residues discovered near the $\mathrm{N}$ terminus. The essential roles of chemokines in biology are mediated through their combinatorial interaction with $\mathrm{G}$ protein-coupled chemokine receptors [4]. Early studies on chemokines were primarily focused on their roles in inflammation or infection responses, they may act as mediators involving in the recruitment of various leukocyte populations, including neutrophils, lymphocytes, monocytes and eosinophils to damaged tissue site [5]. However, increasing evidence revealed that various chemokines may play a critical role in many aspects of tumors, including growth, angiogenesis, metastasis and neutrophils infiltration, through their roles in tumor-promoting inflammation response [6].

CXCL1, also known as GRO-alpha, is a member of ELR + CXC chemokine subgroups characterized by the presence of a conserved glutamic acid-leucine-arginine (ELR) motif [7]. CXCL1-related functions are regulated through its binding to CXCR2 receptor [8]. To date, expanding evidence demonstrated that CXCL1 and CXCR2 are frequently upregulated expression in a variety of human cancers [7, 9-11], and CXCL1 may serve as a source of autocrine/paracrine signal for the malignant process in tumor microenvironment $[12,13]$. For example, it has been reported that silencing of CXCL1 resulted in a significant reduction in tumor proliferation through its effect on apoptosis induction in HCC, suggesting a network of autocrine signal may be involved in CXCL1-associated tumor biology [14]. Furthermore, tumor cells with a rather high level of CXCL1 may have the ability to trigger the infiltration of tumor-associated macrophages (TAMs) and cancer-associated fibroblasts (CAF) into tumor microenvironment, CXCL1 derived from TAMs/CAF can provide a favourable role in regulating intercellular adhesion and crosstalk among tumors cells and TAMs/CAF, and further resulted in an increased in tumor proliferation by a paracrine manner [15].

In this study, we set out to detect the expression pattern of CXCL1 protein in a commercial uterine cervical tissue microarray, in addition to the its relationship with clinical characteristics, and explore the potential exogenous, autocrine as well as paracrine mechanism involving in CXCL1-mediated UCC tumor biolog, possibly via MAPK/ERK-dependent signal pathway.

\section{Materials And Methods}

\section{Immunohistochemistry in a tissue microarray}

A commercial uterine cervical tissue microarray (Alenabio, China), consisting of 40 cases of cancer tissues and 40 of non-cancerous tissues, was used to evaluate CXCL1 protein expression. Of the cancer tissues, 23 patients aged from 30 to 50 , and 17 aged from 51 to 70 . The patients were classified into 3 clinical stages, comprising 4 cases of stage I, 17 of stage II and 19 of stage III. Moreover, tumor 
infiltration was distinguished with scores increasing from T1 to T3, including 34 with grade 1, 5 with grade 2 and 1 with grade 3 . Immunohistochemical staining was conducted as described previously [16]. Protein expression was determined using a primary antibody targeting CXCL1 (1:100; Abcam, USA), which was further matched with a HRP-linked second antibody (Boster Biological Technology, China) at the dilution of 1:200. Immunoreactivity was graded according to the following two parameters, staining intensity and percentage of positive cells. Staining intensity was scored with a value of 1 (weak), 2 (moderate), 3 (strong) and 4 (super strong), and percentage of positive cells was scored with a value of 1 $(<25 \%), 2(25-50 \%), 3(50-75 \%)$ and $4(>75 \%)$. The total score for CXCL1 staining was calculated as the sum of the score of both parameters.

\section{Cell lines, cell culture and cell transfection}

HeLa human cervical cancer cells and PHM1-41 human immortalized cervical stromal cells were obtained from American type culture collection (ATCC, USA). HeLa and PHM1-41 were, respectively, cultured in RPMI-1640 and Dulbecco's Modified Eagle's Medium (DMEM; Gibco, USA), which were supplemented with $10 \%$ fetal bovine serum (FBS; Gibco, USA) containing antibiotics (100 units/ml penicillin and $100 \mathrm{mg} / \mathrm{ml}$ streptomycin) in a $37^{\circ} \mathrm{C}$ humidified incubator with $5 \% \mathrm{CO}_{2}$. To overexpress CXCL1 gene in HeLa and PHM1-41 cells, full-length CDNA of human CXCL1 was subcloned into pcDNA3.1 vectors and the specific vectors targeting cells were ransfected using a lipofectamine 3000 kit (Invitrogen, USA) according to the supplier's instructions. Finally, the transfection efficiency of CXCL1 in HeLa and PHM1-41 cells were tested using ELISA assays.

\section{ELISA analysis}

The culture supernatant derived from cell medium was collected and CXCL1 secretory protein was determined using a Human CXCL1 ELISA Kit (Boster Biological Technology, China) following the manufacturer's protocol. Briefly, a total of $100 \mu$ of culture supernatant as well as different concentrations of recombinant CXCL1 standard protein was added to each well followed by incubating the plate at $37^{\circ} \mathrm{C}$ for $90 \mathrm{~min}$. Following triple rinsing with washing buffer, the plate was coated with 100 $\mu$ biotin-conjugated primary antibody against CXCL1 prior to incubation at $37^{\circ} \mathrm{C}$ for $60 \mathrm{~min}$. After rinsing three times, the plate was coated with $100 \mu \mathrm{l}$ of working fluid and further incubated at $37^{\circ} \mathrm{C}$ for $30 \mathrm{~min}$. Upon the formation of immunocomplex, the plate was washed five times, and $100 \mu \mathrm{l}$ of TMB developer solution was placed into each well for colorimetric determination. After reaction at $37^{\circ} \mathrm{C}$ for 20 min, absorbance of each well was immediately measured at $450 \mathrm{~nm}$ using a spectrophotometer (BioTek Instruments, USA). CXCL1 protein level in culture supernatant was quantified and analyzed based on a standard curve constructed by the absorbance of CXCL1 standard protein.

\section{Cell proliferation detection}

Three separate experiments were employed to evaluate HeLa cell viability. (I) Exogenous effect: HeLa cells at a density of $2 \times 10^{3}$ per well were seed into each well of 96-well plates supplemented with $100 \mu \mathrm{l}$ of medium containing various concentrations of human recombinant CXCL1 (PeproTech, USA). (II) 
Autocrine effect: The same number of HeLa cells overexpressing CXCL1 and their mock controls as described in experiment I were grown in 96-well plates, $100 \mu \mathrm{l}$ of medium supplemented with or without the ERK1/2 inhibitor PD98059 (Sigma, USA) was added into each well. (III) Paracrine effect: Firstly, the conditioned medium (CM) was prepared as follows: CXCL1-overexpressing PHM1-41 cells and their mock controls were inoculated in 100-mm diameter dishes at a density of $5 \times 10^{6}$ cells per $1000 \mu$ of medium. After incubation at $37^{\circ} \mathrm{C}$ for $12 \mathrm{~h}$, the culture supernatant was harvested by centrifugation, and $\mathrm{CM}$ from PHM1-41 cells was produced by addition of different proportions of the supernatant into complete medium. In the paracrine experiment, HeLa cells at a density of $2 \times 10^{3}$ per well were seeded into 96 -well plates, and each well was incubated with $100 \mu \mathrm{l}$ of various proportions of $\mathrm{CM}$. The cells in the above three groups were incubated at $37^{\circ} \mathrm{C}$ for $24 \mathrm{~h}, 48 \mathrm{~h}$ and $72 \mathrm{~h}$, cell viability was thereafter addressed using the Cell Counting Kit-8 (CCK-8) assay.

\section{Cell migration assay}

Consistent with proliferation assay, three experiments for migration assay were as follows. (I) Exogenous effect: The same number of HeLa cells at a density of $2 \times 10^{3}$ were seeded in the top chamber in $100 \mu \mathrm{l}$ serum-free medium, the low chamber was coated with $600 \mu \mathrm{l}$ serum-supplemented medium supplemented with various concentrations of human recombinant CXCL1. (II) Autocrine effect: $2 \times 10^{4}$ of CXCL1-overexpressing HeLa cells and their mock controls in $100 \mu \mathrm{l}$ serum-free medium containing with or without the ERK inhibitor PD98059 were seeded in top chamber, whereas $600 \mu \mathrm{l}$ medium with $10 \% \mathrm{FBS}$ was placed in the low chamber for chemoattraction. (III) Paracrine effect: HeLa cells were maintained in the upper chamber as described in experiment $\mathrm{I}$, and $600 \mu \mathrm{l}$ serum-supplemented medium containing various proportions of $\mathrm{CM}$ was placed in the low chamber. $24 \mathrm{~h}$ after migration, the cells that had attached on the bottom surface of the transwell filters were fixed and stained with methanol/crystal violet solution.

\section{Cell apoptosis assay}

Cell apoptosis was determined by using a commercial apoptotic-Hoechst Staining Kit (Beyotime, Shanghai, China) according to the manufacturer' instructions. Following detection with a confocal microscope at $350 \mathrm{~nm}$, cancer cell apoptosis rate was estimated by the following formula: Apoptosis rate $(\%)=$ apoptosis cell numbers/ total cell numbers $\times 100 \%$.

\section{Western blotting analysis}

Total cell lysates were prepared from cell pellets using RIPA buffer (Solarbio, China) following the manufacturer's protocol. After quantification of protein concentration, a total of $40 \mu \mathrm{g}$ of protein extracts was subjected to western blotting analysis. Different primary antibodies, including anti-p-ERK $(1: 1,000$ dilution; ImmunoWay, USA), anti- ERK (1:1,000 dilution; ImmunoWay, USA), anti-cyclin D1 (1:5,000 dilution; Abcam, USA), anti-Bax (1:1,000; OriGene, China) and anti- $\beta$-actin (1:1,000 dilution; Boster, China) antibodies, were utilized in the analysis, followed by incubation with various HRP-conjugated second antibodies (Boster Biological Technology, China). Immune complexes were visualized using an Enhanced 
Chemiluminescence (ECL) kit (Thermo Fisher Scientific, USA), and the band intensities were calculated using a Gel imaging system (Tanon, China).

\section{Statistical analysis}

All assays were done at least three times, SPSS 20.0 software was applied for statistical analyses. All values were expressed as mean value with their standard deviation, statistical difference between two groups was determined by analysis of variance followed by the Student's t-test. Statistically significant was defined as a P-value $<0.05$.

\section{Results}

\section{CXCL1 is highly expressed in tissues with UCC}

To assess the clinical significance of CXCL1 in UCC, the immunoreactivity of CXCL1 was explored in a uterine cervical tissue microarray. The results from immunohistochemistry assay demonstrated that the expression level of CXCL1 protein was surprisingly increased in tissues with UCC, as compared with that in non-cancerous tissues (Fig. 1). Of the UCC tissues, it was further demonstrated that the upregulation of CXCL1 was extremely correlated with poor clinical stages due to the increased identification of CXCL1 in tissues with stage III. However, this study did not reveal a closer connection of CXCL1 expression with other clinical characteristics including tumor infiltration and patient age (Table 1).

\section{Exogenous CXCL1 contributes to growth, migration and apoptosis of UCC cells}

To functionally determine the roles of CXCL1 in UCC, exogenous CXCL1 was firstly used as a mediator to investigate its potential to the growth, migration and apoptosis of HeLa cells. After 24,48 and $72 \mathrm{~h}$ incubation, the findings from CCK-8 assay indicated that exposure to exogenous CXCL1 (10, 20 or $40 \mathrm{ng} / \mathrm{ml}$ ) induced a obviously dose-dependent increase in HeLa cell growth ability (Fig. 2a). Accordingly, exogenous administration of CXCL1 at concentration of 10,20 or $40 \mathrm{ng} / \mathrm{ml}$ also obviously enhanced HeLa cells migration ability (Fig. 2b and c). Inversely, CXCL1 at concentration of 20 or $40 \mathrm{ng} / \mathrm{ml}$ resulted in the inhibition of apoptosis in HeLa cells (Fig. 2d).

\section{CXCL1 overexpression enhances malignant phenotypes of UCC cells via a autocrine manner}

Since CXCL1 was expressed by uterine cervical tisssues, we therefore intended to explore its expression in HeLa cells. ELISA assays displayed that CXCL1 protein were detected in HeLa cell culture supernatant (Fig. 3a), suggesting that HeLa cells express endogenous CXCL1, which might be further transported into the extracellular fluid as a secretory protein. To adress whether a autocrine mechanism is involved in CXCL1-mediated malignant processes in UCC, HeLa cells overexpressing CXCL1 were constructed by gene transfection. The results showed secretory CXCL1 protein was prominently elevated in CXCL1overexpressing cells relative to their mock controls (Fig. 3A), indicating that stable HeLa cells with expression of CXCL1 were constructed as expected. Additionally, CCK-8 and transwell assays 
demonstrated that CXCL1 overexpression in HeLa cells resulted in obvious increase in cell growth (Fig. 3b) and migration (Fig. 3c and d), as well as decrease in cell apoptosis (Fig. 3e).

\section{Stromal cell-derived CXCL1 facilitates UCC cell malignant processes via a paracrine manner}

To gain further insights into the potential paracrine mechanism involving the roles of CXCL1, PHM1-41 cells overexpressing CXCL1 and their mock controls were established. After verification of the transfection efficiency by using ELISA assay (Fig. 4a), CM was prepared by mixing supernatant from PHM1-41 cell medium with complete medium in various proportions. Subsequently, CCK-8 and transwell assays elucidated that treatment of HeLa cells with CM derived from CXCL1-overexpressing PHM1-41 cells at different proportions obviously enhanced cell growth (Fig. 4b) and migration (Fig. 4c and d) abilities in a proportion dependent manner. Moreover, we also performed apoptosis assay with increasing proportion of PHM1-41 cell-derived CM and observed that the apoptosis rate was obviously inhibited after HeLa cell treatment with $60 \%$ of CXCL1-overexpressing PHM1-41 cell CM (Fig. 4e).

\section{ERK signal regulates UCC cell malignant processes through its direct effect on survival-associated gene}

Increasing evidence has indicated that numerous chemokines are critical for the tumor-specific processes by activating several signaling pathways, especially the MAPK/ERK. Therefore, in the current study, the roles of CXCL1 in regulating the expression of ERK signal-related genes were explored. A predominant increase in the expression of ERK, pERK and cyclin D1, and a marked decrease in the expression of Bax was detected in CXCL1-overexpressing HeLa cells compared with their mock controls by western blotting (Fig. 5a-C). To sought for the underlying mechanism through which ERK signal regulates the roles of CXCL1 in UCC, the malignant behaviors of HeLa cells was further assessed in the presence and absence of ERK inhibitor PD98059, and the rates of proliferation and migration inhibition were commented as follows: inhibition rate $(\%)=$ (the index of cells treatment without PD98059- the index of cells treatment with PD98059) / the index of cells treatment without PD $98059 \times 100 \%$. Our results showed that the growth (Fig. 5d) and migration (Fig. 5e and f) abilities of the CXCL1-overexpressing HeLa cells were obviously reduced to significant levels as compared with their mock controls in the presence of ERK inhibitor PD98059, suggesting the capacity of PD98059 to interrupt CXCL1-mediated UCC tumor processes.

\section{Discussion}

Human CXCL1 belongs to a member of ELR + CXC chemokines, which now contain the epithelial neutrophil-activating protein-78 (ENA-78/CXCL5), granulocyte chemotactic peptide-2 (GCP-2/XCL6), neutrophil-activating peptide-2 (NAP-2/CXCL7), interleukin-8 (IL-8/CXCL8) and growth-related oncogene (GRO) chemokines, in which there are three homologs consisting of GRO a, $\beta$ and $\gamma$, also defined as CXCL1, CXCL2 and CXCL3, respectively [17]. The biological activities of ELR + CXC chemokines were primarily mediated by their interaction with the same receptor, namely CXCR2 [18]. Recently, it is widely acknowledged that several members of the ELR + CXC chemokines have been shown to be constitutively expressed in various types of human cancers, and their overexpression may be implicated in multiple 
aspects of cancer progression including tumor cell proliferation, survival, migration and invasion [16, 1921]. Moreover, ELR $+C X C$ chemokines class has also been proposed to has important potential to promote angiogenesis, a vital biological processes, upon which tumors depend for their malignant behaviors $[22,23]$. Studies on the mechanisms indicated that the involvement of ELR + CXC chemokines in tumor progression was depended on the activation of several signal pathways, such as p38 MAPK/ERK, TAK1/NFKB and Snail/E-cadherin [24-26].

In the current study, we demonstrated that the expression level of CXCL1 in tissues with UCC was elevated dramatically, and its higher expression was extremely associate with poor clinical stages. Consistent with this, it has been suggested that CXCL1 expression was frequently upregulated in several human tumors, such as melanoma, breast and bladder cancers, and increased expression pattern of CXCL1 was predominantly relevant to several clinicopathological features including high grade, advanced stage and positive invasion in bladder cancer in vivo [28-30].

In addition, many studies have also highlighted the crucial roles of CXCL1 in cancer development and progression. For example, it was revealed that secreted CXCL1 or CXCL10verexpression in bladder cancer and melanoma was correlated with enhanced proliferative and metastatic properties for cancer cells, and CXCL1 in vitro was sufficient for the ability of cancer cells to generate tumors in nude mouse xenograft model $[27,30]$. In order to evaluate the potential roles of CXCL1 to contribute to UCC biology, we employed recombinant CXCL1, CXCL1-overexpressing HeLa and PHM1-41 cells for focusing on the effects of CXCL1 on Hela tumor cell malignant behaviors. Our data revealed that CXCL1 recombinants and CXCL1 overexpression effectively promoted cell proliferation and migration properties via both exogenous and autocrine manners.

There is general agreement that the tumor microenvironment is comprised of cancer cells, extracellular matrix and various types of stromal cell subsets, which contain fibroblasts, vessel cells (composed of endothelial cells, smooth muscle cells and pericytes), and diverse inflammatory leukocytes (including neutrophils, macrophages, dendritic cells, mast cells and lymphocytes) [31]. To date, people have gained a deeper understanding of the crosstalk among these cells types which contributes to the formation of tumor-specific microenvironment and further facilitates the oncogenic process via a paracrine loop involving the interaction of chemokines with their corresponding receptors [15]. In thyroid cancer, tumor cell-derived soluble factors facilitated in vitro mast cell activation and recruitment, and the mast cellproduced mediators, especially CXCL1, most effectively enhanced growth, survival and invasion of cancer cells [32]. In addition, in breast cancer, CXCL1 has the ability to attract $\mathrm{CD} 11 \mathrm{~b}^{+} \mathrm{Gr} 1^{+}$myeloid stromal cell population into the tumor microenvironment, which in turn generates several members of chemokines that can serve as paracrine stimulators for the survival of tumor cell [13]. In study demonstrated here, we employed gene transfection to establish PHM1-41 cervical stromal cells overexpressing CXCL1, which can secrete a respectably high level of CXCL1 into medium, and the supernatants derived from medium of CXCL1 overexpressing PHM1-41cell can behave as a promoting mediator for the growth and migration of HeLa cells, indicating that a CXCL1 paracrine manner plays a crucial role in tumor-associated malignant behaviors. Coupled with this, our laboratory also previously 
revealed the critical CXCL3-paracrine mechanism, by which supernatants derived from stromal cells overexpressing CXCL3 raised the abilities of HeLa cell growth and migration [33].

In the current study, we finally revealed that CXCL1 is the crucial chemokine that enhances UCC development by regulating extracellular signal-regulated kinase (ERK) signal-related genes, including cyclin D1, ERK, p-ERK and Bax. ERK signaling is an essential transduction pathway and has received considerable attention because of their important effects on regulating various physiological and pathological processes. It has been suggested that activation of ERK signaling pathway may stimulate tumor cells proliferation through its ability to initiate expression of crucial growth-stimulating genes, such as cyclin D1 and early growth response-1(EGR-1) [34, 35]. CXCL1/CXCR2 axis has been shown to participate in regulation of extracellular signal-regulated kinase 1/2 (ERK1/2) phosphorylation in esophageal cancer, which is responsible for the transcription of EGR-1 gene, the crosstalk of among CXCL1/CXCR2 axis, ERK1/2 and EGR-1 will eventually result in a rapid increase in tumor cell proliferation [36]. Apart from its impact on cell proliferation, ERK signaling is also closely related to regulate the expression of many other genes responsible for anti-apoptosis, angiogenesis and metastasis. In the current study, we confirmed that exogenous administration and overexpression of CXCL1 can effectively inhibit apoptosis of HeLa cells, and result in a decrease in Bax gene expression. Apoptosis, or programmed cell death (PCD), is a controlled form of cell death which allows body to control precisely the number of cells in tissues and is mainly regulated by pro- and anti-apoptotic BCL-2 family members, in which Bax is known as one of the most important mediators to induce the process of apoptosis [37]. It has been increasingly recognized that apoptosis in many types of cancers was frequently inhibited, which at least associated with low expression of pro-apoptotic genes, such as Bax, Bak, Bad and Bcl-Xs, indicating that disruption of the balance between pro- and anti-apoptosis is critical for numerous human tumor processes [37].

\section{Conclusions}

In summary, the current study demonstrated that CXCL1 expression was increased in tissues with UCC, and its higher expression was predominantly correlated with poor clinical stages. In vitro, CXCL1 may contribute the UCC cell malignant behaviors through exogenous, autocrine and paracrine manners, suggesting CXCL1 may be a potential diagnostic marker or therapeutic target in UCC.

\section{Abbreviations}

UCC: uterine cervix cancer; CXCL1:C-X-C motif chemokine 1; HPV: human papillomavirus; TAMs: tumorassociated macrophages; CAF: cancer-associated fibroblasts; ERK: extracellular regulated protein kinase; EGR-1: early growth response-1; Bax: Bcl-2 Associated X Protein.

\section{Declarations}

\section{Author details}


${ }^{1}$ Department of Oncologic Gynecology, The First Hospital of Jilin University, Changchun, Jilin, 130021, People's Republic of China. ${ }^{2}$ Department of Geriatrics, The First hospital of Jilin University, Changchun, Jilin, 130021, People's Republic of China. ${ }^{3}$ Department of Echocardiography, The First hospital of Jilin University, Changchun, Jilin, 130021, People's Republic of China. ${ }^{4}$ Department of Plastic Surgery, The First Hospital of Jilin University, Changchun, Jilin, 130021, People's Republic of China. ${ }^{5}$ Department of Cardiac Surgery, The First Hospital of Jilin University, Changchun, Jilin, 130021, People's Republic of China.

\section{Authors' contributions}

BGW and HJJ designed the experiments and revised the paper; XXM and XLY Performed the experiments and wrote the paper; ZTW analyzed the data; YYT performed immunohistochemical experiments; WYL performed cell experiments. All authors read and approved the final manuscript.

\section{Acknowledgements}

Not applicable.

\section{Competing interests}

Authors have declared that no competing interest exists.

\section{Availability of data and materials}

The data of this manuscript have been presented in the main paper.

\section{Consent for publication}

Not applicable.

\section{Ethics approval and consent to participate}

The study was approved by the Ethics Committee of the Jilin University.

\section{Funding}

The study was supported by grants from Special health project of the Department of Finance of Jilin Province (no. JLSCZD2019-078) and Youth backbone training project of Health Committee of Jilin Province (no. 2017Q018).

\section{References}

1. Bray F, Ferlay J, Soerjomataram I, Siegel RL, Torre LA, Jemal A. Global Cancer Statistics 2018: GLOBOCAN Estimates of Incidence and Mortality Worldwide for 36 Cancers in 185 Countries. CA 
Cancer J Clin. 2108; 68(6): 394-424.

2. Ali-Risasi C, Verdonck K, Padalko E, Vanden Broeck D, Praet M. Prevalence and risk factors for cancer of the uterine cervix among women living in Kinshasa, the Democratic Republic of the Congo: a cross-sectional study. Infect Agent Cancer. 2015;10:20.

3. Reis Campos LM, Luz Dias Fd, Antunes LM, Murta EF. Prevalence of micronuclei in exfoliated uterine cervical cells from patients with risk factors for cervical cancer. Sao Paulo Med J. 2008;126(6):3238.

4. Vicari AP, Caux C. Chemokines in cancer. Cytokine Growth Factor Rev. 2002;13(2):143-54.

5. Lukacs NW, Hogaboam CM, Kunkel SL, Chensue SW, Burdick MD, Evanoff HL, Strieter RM. Mast cells produce ENA-78, which can function as a potent neutrophil chemoattractant during allergic airway inflammation. J Leukoc Biol. 1998;63(6):746-51.

6. Rollins BJ. Inflammatory chemokines in cancer growth and progression. Eur J Cancer. 2006;42(6):760-7.

7. O'Hayer KM, Brady DC, Counter CM. ELR + CXC chemokines and oncogenic Ras-mediated tumorigenesis. Carcinogenesis. 2009;30(11):1841-7.

8. Lázár-Molnár E, Hegyesi H, Tóth S, Falus A. Autocrine and paracrine regulation by cytokines and growth factors in melanoma. Cytokine. 2000;12(6):547-54.

9. Cao Z, Fu B, Deng B, Zeng Y, Wan X, Qu L. Overex-pression of Chemokine (C-X-C) ligand 1 (CXCL1) associated with tumor progression and poor prognosis in hepatocellular carcinoma. Cancer Cell Int. 2014;14(1):86.

10. Wei ZW, Xia GK, Wu Y, Chen W, Xiang Z, Schwarz RE, et al. CXCL1 promotes tumor growth through VEGF pathway activation and is associated with inferior survival in gastric cancer. Cancer Lett. 2015;359(2):335-43.

11. Miyake M, Lawton A, Goodison S, Urquidi V, Rosser CJ. Chemokine (C-X-C motif) ligand 1 (CXCL1) protein expression is increased in high-grade prostate cancer. Pathol Res Pract. 2014;210(2):74-8.

12. Cheng WL, Wang CS, Huang YH, Tsai MM, Liang Y, Lin KH. Overexpression of CXCL1 and its receptor CXCR2 promote tumor invasion in gastric cancer. Ann Oncol. 2011;22(10):2267-76.

13. Acharyya S, Oskarsson T, Vanharanta S, Malladi S, Kim J, Morris PG, et al. A CXCL1 paracrine network links cancer chemoresistance and metastasis. Cell. 2012;150(1):165-78.

14. Han KQ, He XQ, Ma MY, Guo XD, Zhang XM, Chen J, et al. Targeted silencing of CXCL1 by siRNA inhibits tumor growth and apoptosis in hepatocellular carcinoma. Int J Oncol. 2015;47(6):2131-40.

15. Miyake M, Hori S, Morizawa Y, Tatsumi Y, Nakai Y, Anai S, et al. CXCL1-Mediated Interaction of Cancer Cells with Tumor-Associated Macrophages and Cancer-Associated Fibroblasts Promotes Tumor Progression in Human Bladder Cancer. Neoplasia. 2016;18(10):636-46.

16. Qi Y, Zhao W, Li M, Shao M, Wang J, Sui H, et al. High C-X-C motif chemokine 5 expression is associated with malignant phenotypes of prostate cancer cells via autocrine and paracrine pathways. Int J Oncol. 2018;53(1):358-70. 
17. Wang D, Wang H, Brown J, Daikoku T, Ning W, Shi Q, et al. CXCL1 induced by prostaglandin E2 promotes angiogenesis in colorectal cancer. J Exp Med. 2006;203(4):941-51.

18. Ahuja SK, Murphy PM. The CXC chemokines growth-regulated oncogene (GRO) alpha, GRObeta, GROgamma, neutrophil-activating peptide-2, and epithelial cell-derived neutrophil-activating peptide78 are potent agonists for the type $B$, but not the type $A$, human interleukin-8 receptor. J Biol Chem. 1996;271(34):20545-50.

19. Gui SL, Teng LC, Wang SQ, Liu S, Lin YL, Zhao XL, et al. Overexpression of CXCL3 can enhance the oncogenic potential of prostate cancer. Int Urol Nephrol. 2016;48(5):701-9.

20. Yuan A, Yang PC, Yu CJ, Chen WJ, Lin FY, Kuo SH, Luh KT. Interleukin-8 messenger ribonucleic acid expression correlates with tumor progression, tumor angiogenesis, patient survival, and timing of relapse in non-small-cell lung cancer. Am J Respir Crit Care Med. 2000;162(5):1957-63.

21. Kim SJ, Uehara H, Karashima T, Mccarty M, Shih N, Fidler IJ. Expression of interleukin-8 correlates with angiogenesis, tumorigenicity, and metastasis of human prostate cancer cells implanted orthotopically in nude mice. Neoplasia. 2001;3(1):33-42.

22. Gijsbers K, Gouwy M, Struyf S, Wuyts A, Proost P, Opdenakker G, et al. GCP-2/CXCL6 synergizes with other endothelial cell-derived chemokines in neutrophil mobilization and is associated with angiogenesis in gastrointestinal tumors. Exp Cell Res. 2005;303(2):331-42.

23. Strieter RM, Polverini PJ, Kunkel SL, Arenberg DA, Burdick MD, Kasper J, et al. The functional role of the ELR motif in CXC chemokine-mediated angiogenesis. J Biol Chem. 1995;270(45):27348-57.

24. Yung MM, Tang HW, Cai PC, Leung TH, Ngu SF, Chan KK. et al. GRO-a and IL-8 enhance ovarian cancer metastatic potential via the CXCR2-mediated TAK1/NFKB signaling cascade. Theranostics. 2018;8(5):1270-85.

25. Yang HT, Cohen P, Rousseau S. IL-1 beta-stimulated activation of ERK $1 / 2$ and p38alpha MAPK mediates the transcriptional up-regulation of IL-6, IL-8 and GRO-alpha in HeLa cells. Cell Signal. 2008;20(2):375-80.

26. Chen L, Pan XW, Huang H, Gao Y, Yang QW, Wang LH, et al. Epithelial-mesenchymal transition induced by GRO-a-CXCR2 promotes bladder cancer recurrence after intravesical chemotherapy. Oncotarget. 2017;8(28):45274-85.

27. Dhawan P, Richmond A. Role of CXCL1 in tumorigenesis of melanoma. J Leukoc Biol. 2002;72(1):918.

28. Block MS, Charbonneau B, Vierkant RA, Fogarty Z, Bamlet WR, Pharoah PD, et al. NF-kappa B genes have a major role in inflammatory breast cancer. BMC Cancer. 2008;8:41.

29. Miyake M, Lawton A, Goodison S, Urquidi V, Gomes-Giacoia E, Zhang G, et al. Chemokine (C-X-C) ligand 1 (CXCL1) protein expression is increased in aggressive bladder cancers. BMC Cancer. 2013;13:322.

30. Kawanishi H, Matsui Y, Ito M, Watanabe J, Takahashi T, Nishizawa K, et al. Secreted CXCL1 is a potential mediator and marker of the tumor invasion of bladder cancer. Clin Cancer Res. 2008;14(9):2579-87. 
31. Raman D, Sobolik-Delmaire T, Richmond A. Richmond. Role of chemokines in tumor growth. Cancer Lett. 2007;256(2):137-65.

32. Melillo RM, Guarino V, Avilla E, Galdiero MR, Liotti F, Prevete N, et al. Mast cells have a protumorigenic role in human thyroid cancer. Oncogene. 2010;29(47):6203-15.

33. Qi YL, Li Y, Man XX, Sui HY, Zhao XL, Zhang PX, et al. CXCL3 overexpression promotes the tumorigenic potential of uterine cervical cancer cells via the MAPK/ERK pathway. J Cell Physiol. 2020;235(5):4756-65.

34. Yang C, Klein EA, Assoian RK, Kazanietz MG. Kazanietz. Heregulin beta1 promotes breast cancer cell proliferation through Rac/ERK-dependent induction of cyclin D1 and p21Cip1. Biochem J. 2008;410(1):167-75.

35. Ma Y, Cheng Q, Ren Z, Xu L, Zhao Y, Sun J, et al. Induction of IGF-1R expression by EGR-1 facilitates the growth of prostate cancer cells. Cancer Lett. 2012;317(2):150-6.

36. Wang B, Hendricks DT, Wamunyokoli F, Parker MI. A growth-related oncogene/CXC chemokine receptor 2 autocrine loop contributes to cellular proliferation in esophageal cancer. Cancer Res. 2006;66(6):3071-7.

37. Zeren T, Inan S, Vatansever HS, Sayhan S. Significance of apoptosis related proteins on malignant transformation of ovarian tumors: A comparison between Bcl-2/Bax ratio and p53 immunoreactivity. Acta Histochem. 2014;116(8):1251-8.

\section{Tables}

Table 1. Immunostaning intensities of CXCL1 protein in a uterine cervix tissue microarray.

\begin{tabular}{cccc}
\hline Clinical characteristic & Subgroup & $\overline{\text { Score }}_{ \pm \mathrm{S} \square}$ & $P$ value \\
\hline Non-cancer & -- & $4.37 \pm 1.48$ & 0.0187 \\
UCC & -- & $5.15 \pm 1.41$ & \\
\hline Clinical stage & I -II & $4.71 \pm 1.42$ & 0.0376 \\
\hline Tumor infiltration & T1 & $5.63 \pm 1.26$ & \\
\hline & TII & $5.00 \pm 1.44$ & 0.1091 \\
\hline Age(years) & $\leq 50$ & $5.30 \pm 1.52$ & 0.4265 \\
\hline
\end{tabular}

\section{Figures}




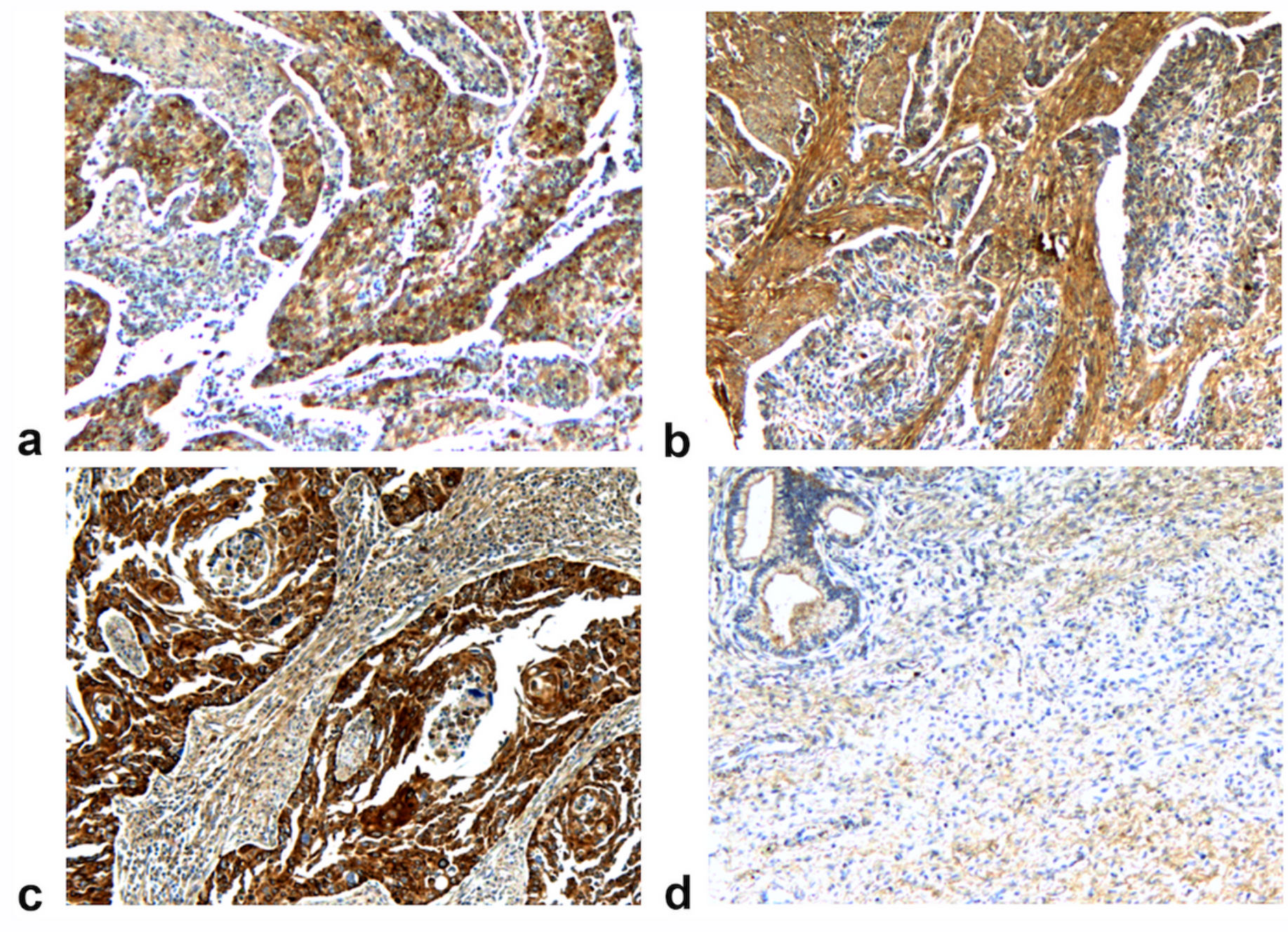

Figure 1

CXCL1 is highly expressed in tissues with UCC. Strong immunohistochemical staining of CXCL1 in tissues with stage I (a), II (b) and III (c), comparing non-cancerous tissues with weak staining (d), was detected in a uterine cervical tissue microarray. 
a
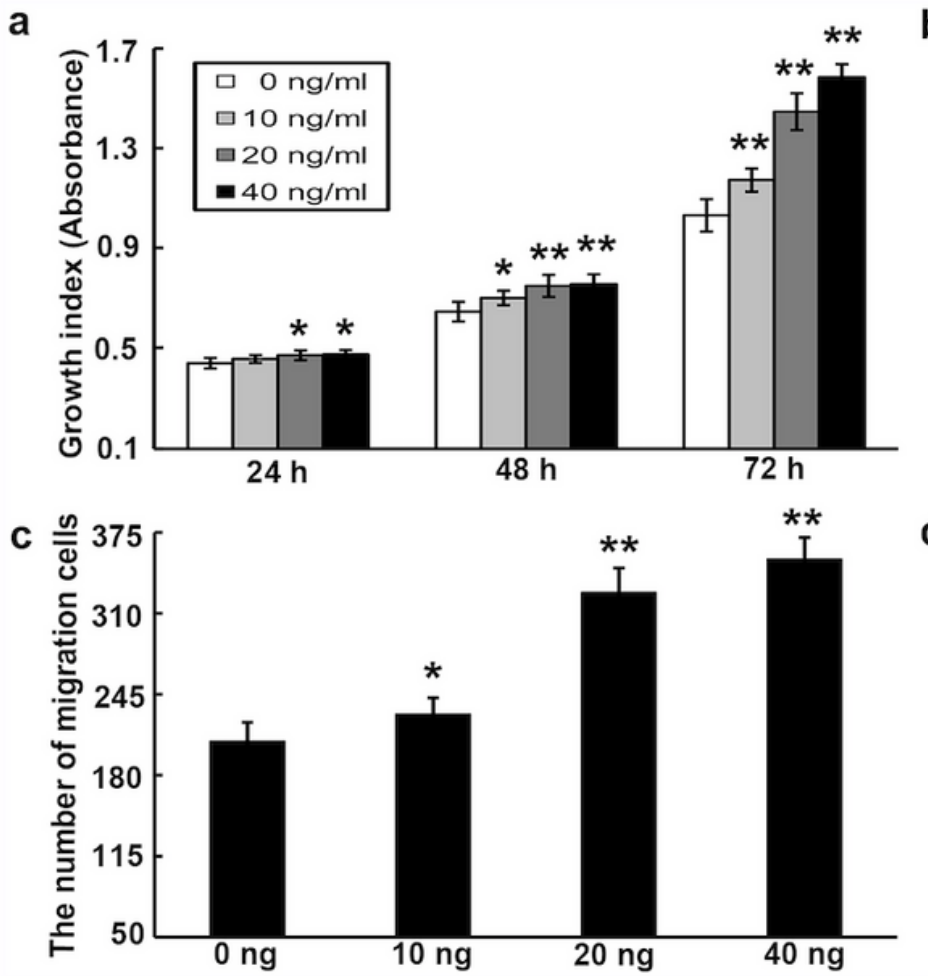

b
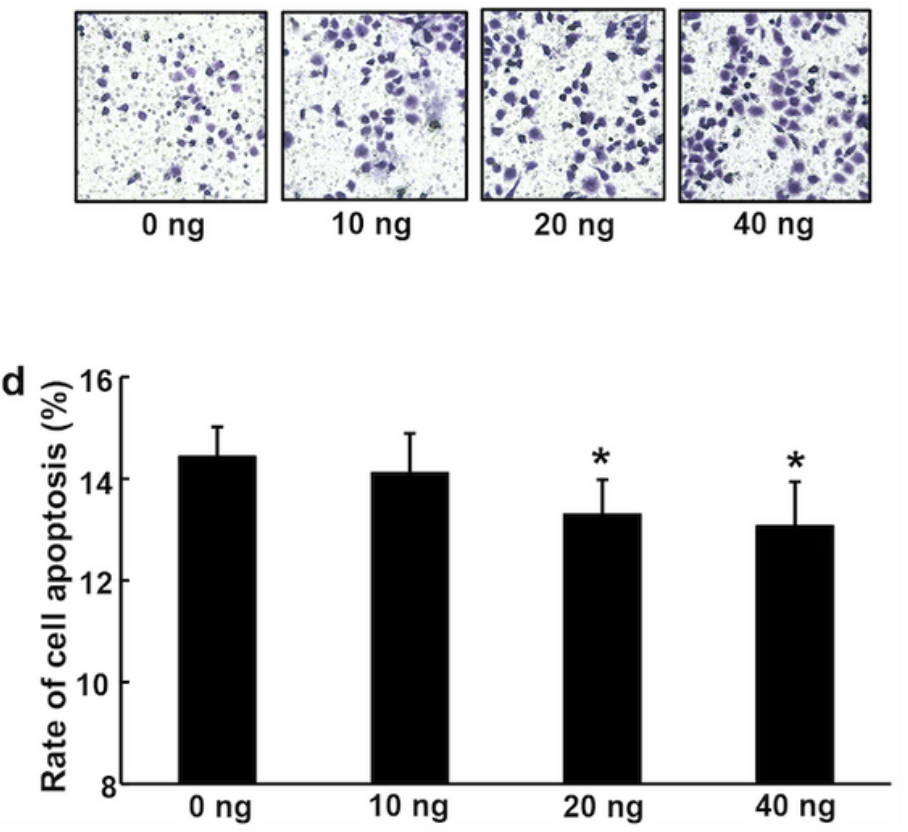

Figure 2

Exogenous CXCL1 facilitates the malignant behaviors of HeLa cells. a The role of different concentrations of exogenous CXCL1 on the proliferation of HeLa cells was tested by CCK-8 assay. b, c Cell migration ability was determined by transwell analysis after HeLa cells treatment with different concentrations of exogenous CXCL1. d HeLa cell apoptosis rate was identified by using the ratio of apoptosis cell numbers to total cell numbers $\left({ }^{*} P<0.05,{ }^{* *} P<0.01\right)$ 


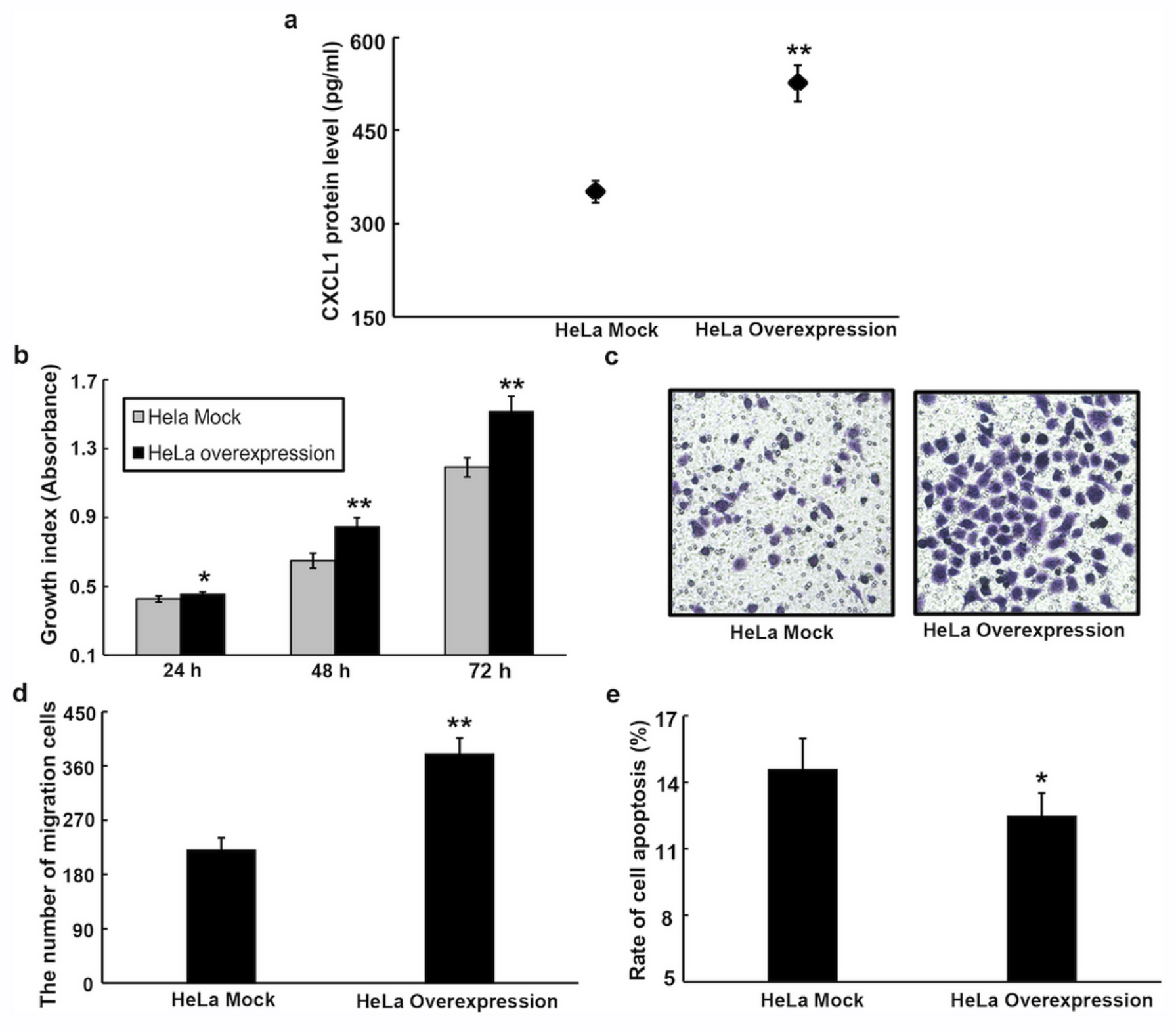

Figure 3

CXCL1 overexpression contributes to proliferation, migration and apoptosis of HeLa cells via a autocrine manner. a The protein expression of CXCL1 in the supernatant derived from HeLa cells overexpressing CXCL1 and their mock controls medium was assessed by ELISA assay; $b$ The proliferation ability of HeLa cells overexpressing CXCL1 was significantly increased as compared to their mock controls; $\mathrm{c}, \mathrm{d}$ The cell migration capacity was notably enhanced after CXCL1 overexpression in HeLa cells; e CXCL1 overexpression led to the inhibition of apoptosis in HeLa cells $\left({ }^{*} P<0.05, * \star P<0.01\right)$ 
a

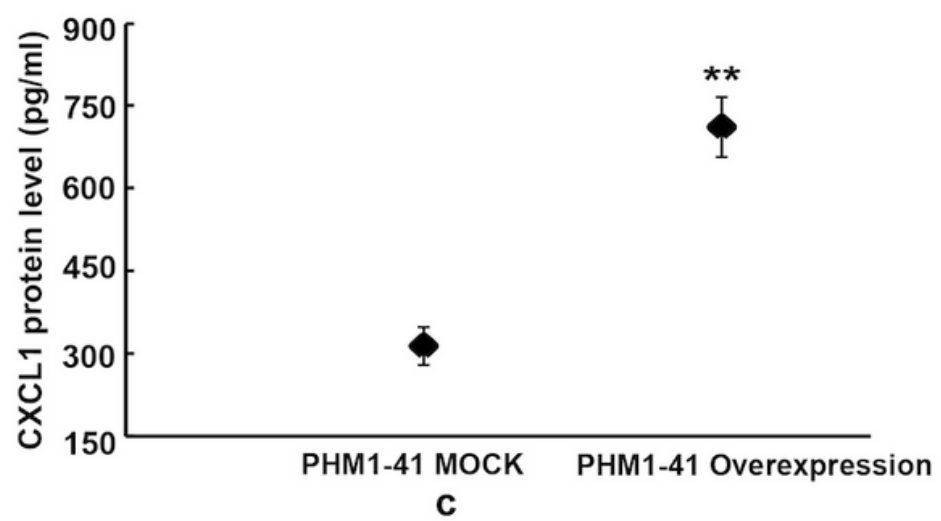

b
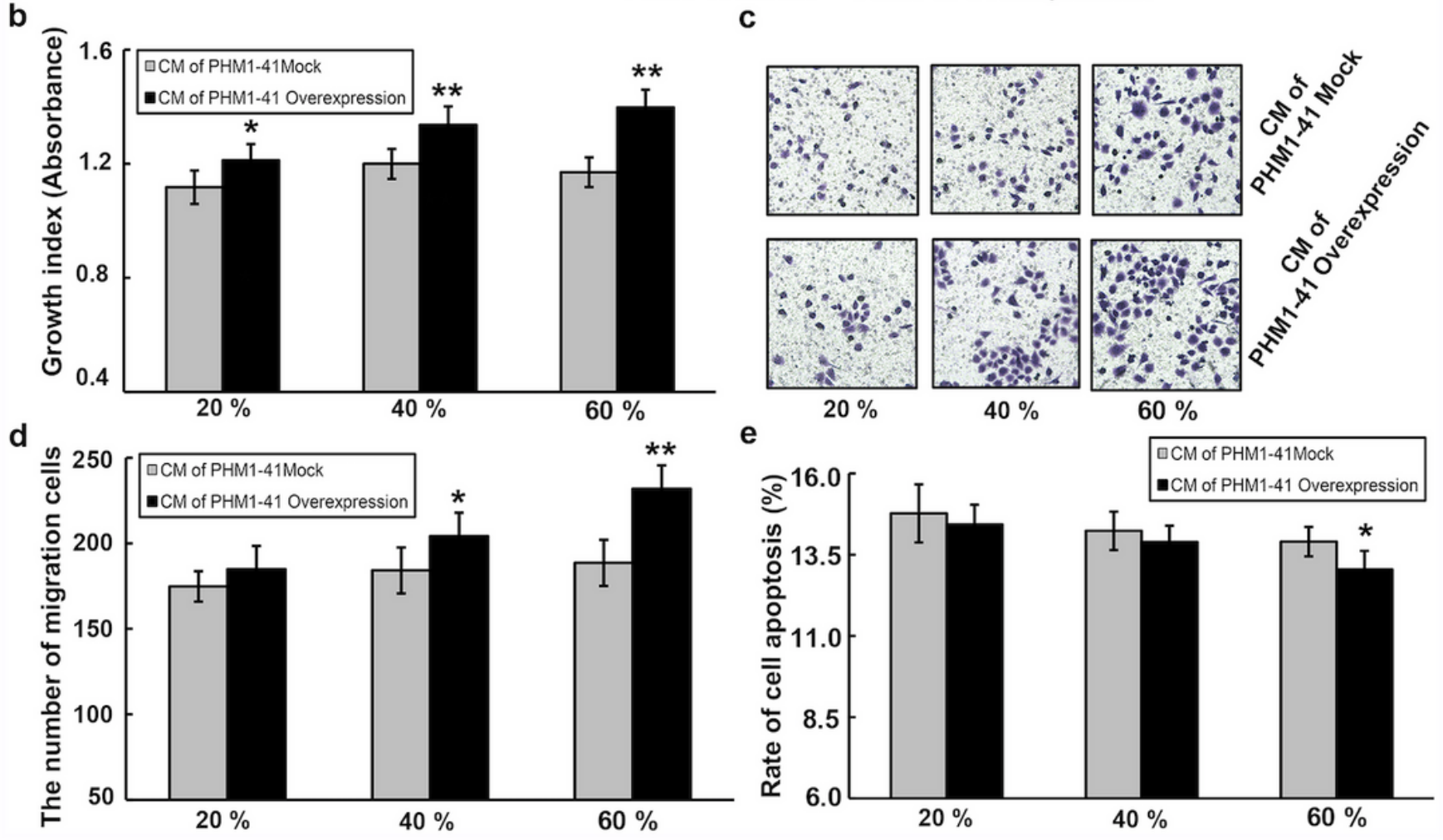

Figure 4

Stromal cell-derived CXCL1 enhances oncogenic potential of HeLa cells via a paracrine manner. a CXCL1 protein level in PHM1-41 cells overexpressing CXCL1 and their mock controls-derived supernatant was determined using ELISA assay; $b$ The growth ability of HeLa cells treatment with CM from PHM1-41 cells overexpressing CXCL1 and their mock controls was detected by CCK-8 assay; c, $d$ The effect of various proportions of CM derived from PHM1-41 cells overexpressing CXCL1 and their mock controls on the HeLa cell migration was explored by transwell analysis; e HeLa cell apoptosis rate was identified after treatment of HeLa cells with various proportions of PHM1-41 cells overexpressing CXCL1 and their mock controls-derived $\mathrm{CM}\left({ }^{*} \mathrm{P}<0.05,{ }^{* \star} \mathrm{P}<0.01\right)$ 
a

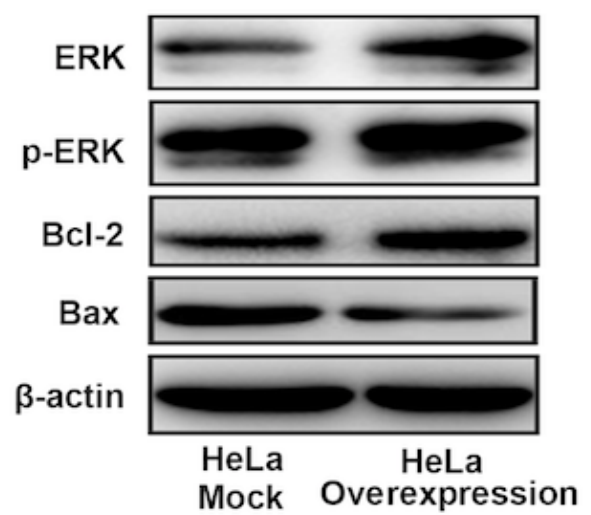

C

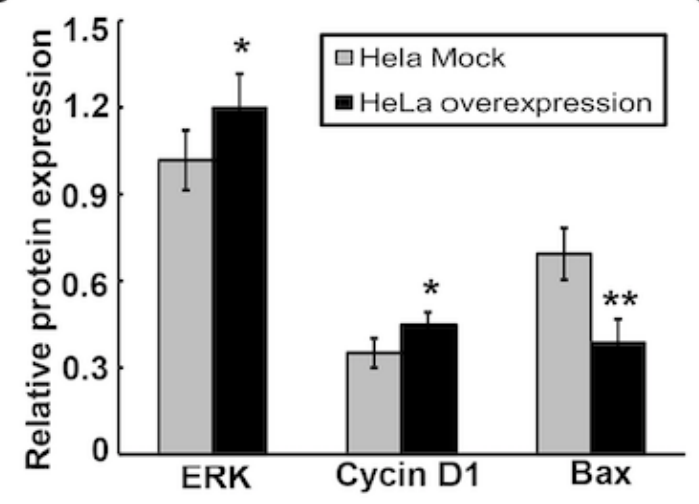

e

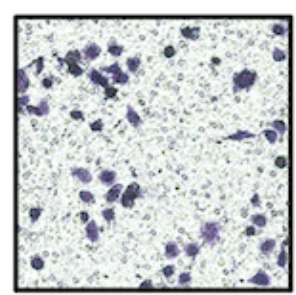

Hela Mock b

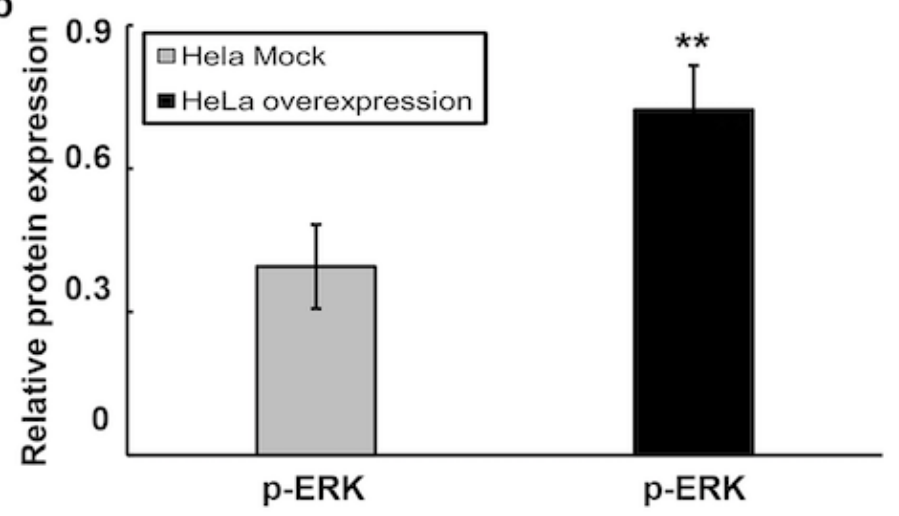

d

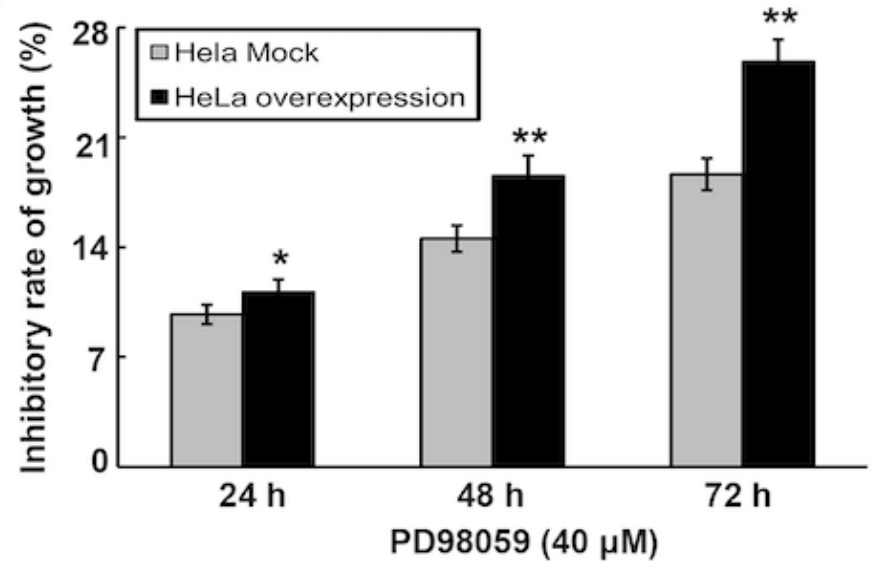

f

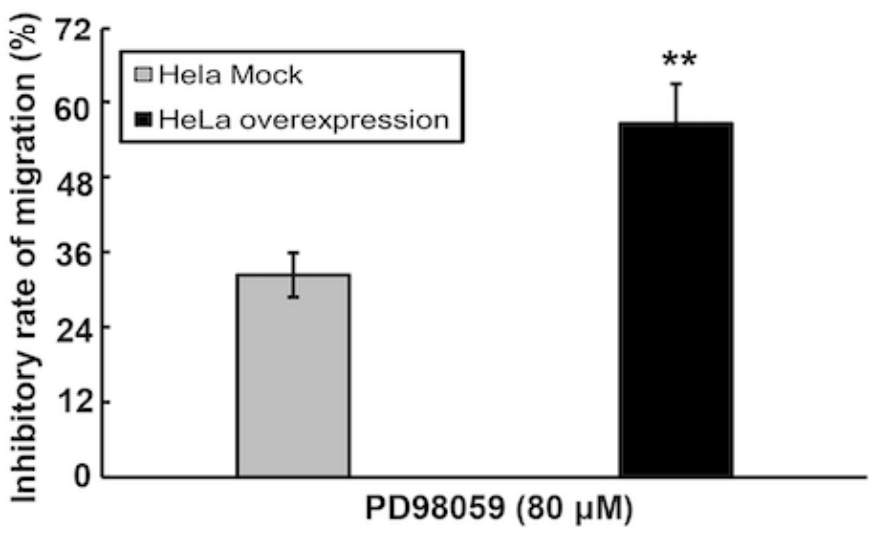

Figure 5

ERK signal involves in CXCL1-mediated malignant phenotypes in HeLa cells. a Expression of ERK, p-ERK, Cyclin D1 and Bax at protein levels from HeLa cells overexpressing CXCL1 and their mock controls were assessed by western blotting; $b$ The protein level of the p-ERK was quantitated and normalized to total ERK from western blotting; c Protein expression of ERK, Cyclin D1 and BAX were statistically analyzed from western blotting, and their bands were normalized to $\beta$-actin; $d$ The proliferation inhibition rate of ERK inhibitor PD98059 on HeLa cells overexpressing CXCL1 and their mock controls was determined by 
CCK-8 assay; $\mathrm{e}, \mathrm{f}$ The effect of ERK inhibitor PD98059 on migration inhibition rate of HeLa cells overexpressing CXCL1 and their mock controls was assessed by transwell analysis $\left({ }^{\star} P<0.05,{ }^{*} P<0.01\right)$ 